\title{
DEVELOPMENTAL INHIBITION IN DROSOPHILA USING DIHYDROXYBENZOIC ACID ISOMERS
}

\author{
W. C. LEVENGOOD ${ }^{1}$ and R. DAMRAUER ${ }^{2}$ \\ ${ }^{1}$ The University of Michigan, Institute of Science and Technology, Ann Arbor, \\ Michigan 48107, and ${ }^{2}$ Chemistry Department, University of Colorado, Denver \\ Center, Denver, Colorado 802202
}

(Received 14 October 1968)

\begin{abstract}
Five isomers of dihydroxybenzoic acid were added to a standard culture medium of Drosophila melanogaster and their effect on development time and progeny yield examined. Each isomer produced a distinct level of inhibitive in:luence throughout continuous inbred generations. The degree of developmental perturbation from a particular isomer was dependent on its protondonating power. Details of the alterations indicated enzyme inhibitions associated with the reaction kinetics of oxidative processes. A simple withdrawal of oxygen by briefly submerging pupae in water also altered the ontogenetic cycle suggesting an analogue of the isomer effect. Lengthened development produced by oxygen withdrawal in first-generation pupae was transferred to succeeding inbred generations. Published pharmacological studies in which drugs were introduced into Drosophila medium are given an interpretation based on the findings with the dihydroxybenzoic acid isomers.
\end{abstract}

\section{INTRODUCTION}

A CONSISTENT reduction in the yield of progeny from Drosophila was previously reported using a culture medium containing a low concentration of protocatechuic acid (Levengood and Shinkre, 1960). Another isomer, gentisic acid, has been definecl as a primary antioxidant or free radical acceptor in oxygen-dependent photodynamic injury (EPSTEIN et al., 1965). The dihydroxyl phenols are also closely coupled with the metabolic pathway in melanogenesis and its associated oxidative processes; consequently, during the development stages of insect metamorphosis the polyphenols play a significant rôle in the hardening and darkening of the cuticle; in fact, 3,4-dihydroxybenzoic acid has been identified (GILmour, 1961) in the hardened secretion forming cockroach oöthecae.

Consideration of these various factors which conclusively demonstrate the importance of the dihydroxybenzoic acid structure in the morphogenetic phases of insect development led to the investigations described here. The possibility of altering the physiological mode of action of the polyphenols was examined by adding a series of dihydroxybenzoic acid isomers to Drosophila melanogaster medium. The influence of these isomers was studied in relation to embryonic development and the yield of progeny. The manner in which the patterns of embryogenesis changed with the proton-donating power or acidity of the isomers 
suggested a possible enzyme inhibition. The developmental data are shown to be in accord with inhibition in a first-order reaction. An examination of published information on the influence of drugs administered to Drosophila also suggested an enzyme inhibition mechanism.

The in vivo antioxidant properties of the isomers also led to a further consideration of a possible perturbation within oxidative processes. A simple method of brief oxygen withdrawal was examined as an independent, non-chemical means of altering oxidation rates. Brief submergence of first-generation pupae in water produced an inherited increase in development time which persisted without further oxygen removal. The fact that oxygen withdrawal perturbs development in somewhat the same manner as the phenols led to the hypothesis that, in both cases, oxidative processes are being altered.

\section{METHOD}

The dihydroxybenzoic acid isomers were added to a regular cornmeal molasses diet using $0.05 \mathrm{~g}$ of each phenol $/ 100 \mathrm{ml}$ of water. This provided a concentration of approximately $2 \cdot 7 \times 10^{-3} \mathrm{M}$ in the final diet. The control cultures were raised on the same diet without the addition of an isomer. Each generation series was initiated on the same date. The medium was placed in half-pint bottles and six pairs of flies were used for each inbred generation. The temperature was maintained at $23^{\circ} \mathrm{C}$ with a $\pm 1^{\circ} \mathrm{C}$ extreme variation. The parents were randomly selected from the previous generation, left in the bottles 10 days, then removed before the new flies emerged. Progeny counts were made 8 days, starting the day on which the new generation of flies emerged. The Oregon-R strain of flies was used throughout these studies and this strain was previously shown to have a development time from the start of a culture to the emergence of the adult flies of $13 \cdot 6 \pm 1 \cdot 12$ days. The extreme values in the development time were 11 and 16 days. This $13 \cdot 6$ day mean is a characteristic of the Oregon-R strain and is not due to overcrowding or larval competition (LEvengood, 1966).

\section{RESULTS}

In Fig. 1 are the progeny values from ten continuous inbred generations raised on the doped medium. It may be seen that each of the isomers exhibits a distinct level of effect on the yield of progeny and there is little overlapping in the various curves. The observed fluctuations occurring from one generation to the next may be expected from the influence of external environmental factors (LEVENGOOD, 1965).

\section{Influence of molecular structures}

A consideration of the stereochemical configurations of these isomers offered at least a qualitative suggestion as to their effects on the levels of progeny shown in the Fig. 1 data. The influence of molecular substituents on the strength of benzoic acid has been quantitatively evaluated by SHORTER and STuBbs (1949). Considering the isomer group used in this study, these authors show that the change in the free 
energy of ionization varies considerably throughout the series. These free energy changes are compared in Table 1 with the mean values of progeny and development time from each isomer. Also listed in Table 1 are the $\mathrm{p} K_{4}$ or acidity values taken from the same source.

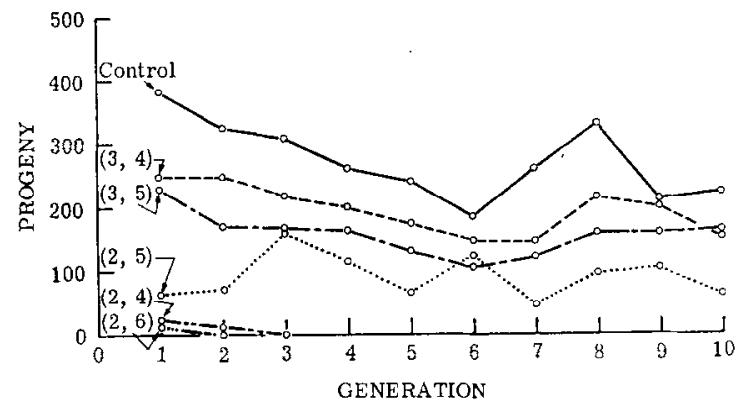

Fig. 1. Progeny yields from ten consecutive inbred generations of $D$. melanogaster fed isomers of dihydroxybenzoic acid.

TABlE 1-MEAN VALUES OF PROGENY AND DEVELOPMENT TIME COMPARED WITH CHANGES IN FREE ENIRGY OF IONIZATION AND ACID STRENGTH OF DIHYDROXYBENZOIC ACID ISOMERS

\begin{tabular}{ccccc}
\hline $\begin{array}{c}\text { Dihydroxybenzoic } \\
\text { acid } \\
\text { isomer }\end{array}$ & $\begin{array}{c}\text { Mean } \\
\text { progeny }\end{array}$ & $\begin{array}{c}\text { Mean } \\
\text { development } \\
\text { time (days) }\end{array}$ & $\begin{array}{c}\text { Changes in free } \\
\text { energy of } \\
\text { ionization* } \\
\Delta\left(-R T \ln K_{A}\right)\end{array}$ & $\begin{array}{c}\text { Acidity* } \\
\mathrm{p} K_{A}\end{array}$ \\
\hline Control & $277 \cdot 3$ & $15 \cdot 6$ & - & - \\
3,4 & $196 \cdot 9$ & $16 \cdot 4$ & +420 & $4 \cdot 48$ \\
3,5 & $158 \cdot 8$ & $16 \cdot 3$ & -180 & $4 \cdot 04$ \\
2,5 & $92 \cdot 5$ & $20 \cdot 2$ & -1640 & $2 \cdot 97$ \\
2,4 & $12 \cdot 0$ & $24 \cdot 0$ & -1190 & $3 \cdot 30$ \\
2,6 & $7 \cdot 5$ & $27 \cdot 0$ & -3920 & $2 \cdot 30$ \\
\hline
\end{tabular}

* Data from Shorter and StubBs (1949): $R$ is the gas constant, $T$ the absolute temperature, anc. $K_{A}$ the association constant.

In general, the data in Table 1 demonstrate that those isomers with the large negative values of free energy of ionization and highest acid strength (low $\mathrm{p} K_{A}$ value) perturb the progeny and development time to the greatest degree. If the $\mathrm{p} K_{A}$ data in Fig. 1 are plotted as a function of progeny and development time reasonably linear relationships are obtained. This suggests the direct involvement of the proton-donating power with the developmental processes.

The hardening and darkening of the insect cuticle involve a scheme in which the polyphenols alter the redox potential of the blood and these reactions do not occur until the ontogenetically correct time (RICHaRDS, 1953). The reduction in free energy of ionization and the increased proton-donating power could alter the in 
vivo redox state providing the isomers become involved in the ontogenetic melanin processes. The structural similarity between the isomers increases the probability of this interaction. The possibility of structurally similar phenol groups being attracted to melanin or a precursor through the process of carboxyl incorporation was suggested in the work of CHEN and CHavin (1966). Thus carboxyl incorporation offers one possible mechanism by which each isomer may become actively involved in the metabolic stages of melanization.

\section{Proposed reaction kinetics}

The systematic variations betwecn progeny and development time listed in Table 1 suggested a deep-seated mechanism, possibly the proton-donating characteristics of the isomers are altering an enzyme system. It was previously shown (LEVENGOOD, 1966) in a study concerning exogenously induced developmental changes in Drosophila that progeny yields may be conveniently taken as representing the final equilibrium concentration of end-products resulting from a perturbed enzyme system. The time to reach this end-product state, the development time, was considered as being directly related to the reaction rate in this perturbed molecular array. For small disturbances in a dynamic system, it is advantageous to consider the rate of reaction as being proportional to the magnitude of the concentrations; that is, the system follows first-order reaction kinetics. The data were examined from this viewpoint and a plot of the log of progeny vs. development time was found to be linear as predicted from this theory.

The alignment of the data in Table 1 was also suggestive of such an inhibition mechanism since the isomers are introducing perturbations in the progeny and development cycles. A plot of the mean values of progeny and the development time is shown in Fig. 2 and the linearity on the semi-logarithmic plot suggests such an enzyme inhibition mechanism in a first-order system (rate constant, $k=$ $\left.0.35 \times 10^{-5} / \mathrm{sec}\right)$.

The induced alterations in progeny and development time listed in Table 1 were produced by continuously subjecting the generation of flies to the medium containing the isomer. To determine how rapidly the development time returned to the normal level, the flies were grown for five generations in regular medium following the ten generations in the chemically treated medium. The control, the 3,4 , the 3,5 , and the 2,5 isomers were examined in this five-generation series. It was found that the 3,4 isomer returned in the first generation on regular medium to the normal range of development time with a mean value of 14.0 days for the five-generation series. The 3,5 isomer was a borderline case with a mean fivegeneration development time of 16.0 days (the development time is considered as being altered if the value is greater than 16 days). The 2,5 isomer, on the other hand, disclosed a decrease in development time but remained at an abnormally high level with a five-generation mean value of 18.6 days. This transference to succeeding generations suggested that the alteration in the enzyme system was essentially more permanent in the case of the 2,5 isomer. The progeny curves in this five-generation series, in contrast with those shown in Fig. 1, disclosed 
considerable overlap or crossing and this indicates a different rate of return to normal conditions within each of the test series. After removal of the chemical from the medium a homeostatic condition may operate to return the perturbed system to the normal level. A cyclic pattern of homeostasis operating through inbred generations has been observed in magnetically perturbed systems (LEVENGOOD, 1967). In the case of the 2,5 isomer, the return rate was of a much lower magnitude than in the 3,5 and 3,4 series.

\section{Related pharmacological studies}

The possibility of inducing genetic effects in Drosophila by adding drugs directly to the normal medium was investigated by LuNING (1966). In these tests barbiturates were added in various concentrations to Drosophila medium (cornmeal, molasses medium of the same type as used in the studies recorded here) and their influence on the progeny and development time examined. In these studies, Luning introduced a given number of eggs (25) into vials containing different concent:ations of the drugs, then he recorded the adults and the length of embryonic and postembryonic development. These drugs were shown to produce different effects on the development time and the final adult progeny.

From the present studies with the dihydroxybenzoic acid isomers and the suggested enzyme inhibition mechanism (Fig. 2), it seemed worth while to examine

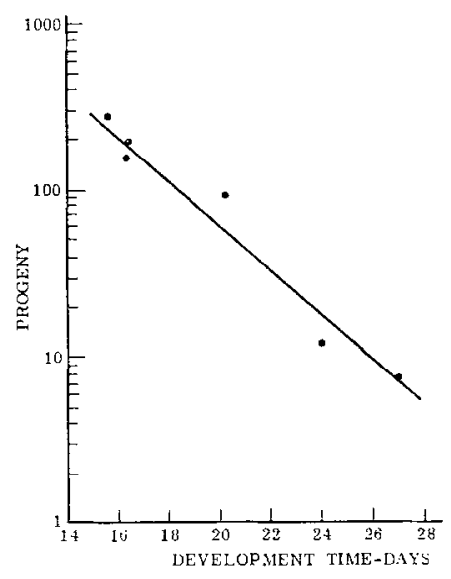

FIG. 2. Relationship between mean values of progeny and development time from isomer-treated series of $D$. melanogaster.

Luning's data from this same viewpoint. From his paper, we chose the data for pentothal sodium, since as a function of concentration this drug appeared to be intermediate in its influence on both the final yield of adults and the alteration of development time. The pentothal sodium data are plotted in Fig. 3 and, although there is evident scatter, a linearity does appear to be evident. It is unfortunate that in this study there was apparently no attempt to examine the influence of the 
chemicals on succeeding generations or the effect of returning the organisms to normal medium.

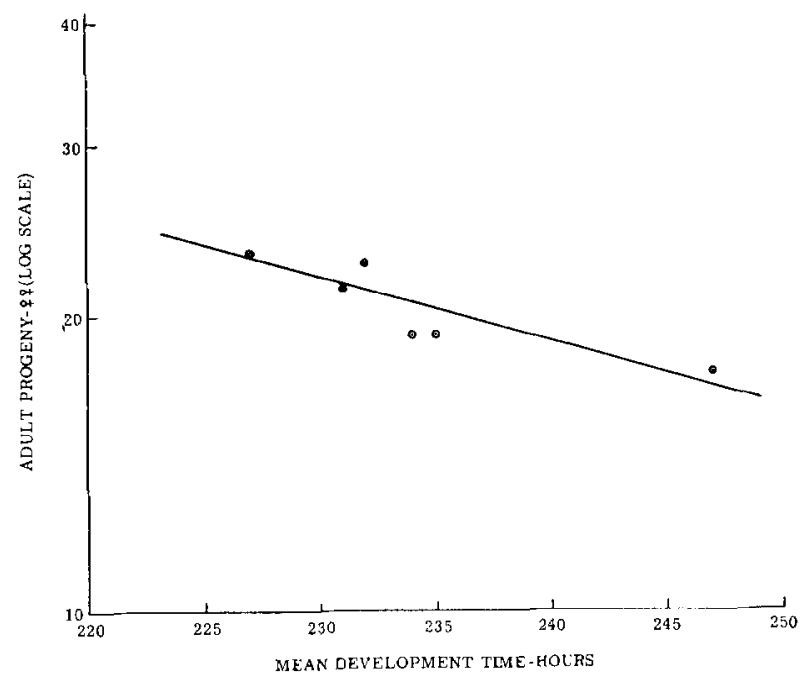

Fig. 3. Relationship between mean values of progeny and development time from D. melanogaster fed pentothal sodium (data from LuNING, 1966).

Although the barbiturates may not be involved in the melanin complex they do appear to influence progeny and development time in a manner analogous to the dihydroxy phenols. The biological effects of barbiturates have been shown by KYOGOKU et al. (1968) to be related to specific hydrogen bonding with adenine derivatives. These workers also point out that lower $\mathrm{p} K_{A}$ values for the barbiturates undoubtedly enhances the strength of this hydrogen bond. The data shown in Fig. 3 may, therefore, represent in vivo perturbations similar to those induced by the phenols (Fig. 2), the common factor related to both sets of data being the proton-donating power of the additive molecules.

Simple interference with oxidative processes

The addition of dihydroxybenzoic acid isomers to the medium and their in viva influence (Table 1) on development time and the yield of progeny suggest that they may be altering oxidative mechanisms in developmental systems. The stereochemical structure of the added isomers are very similar to naturally synthesized phenols and, therefore, may block the pathway or reduce the reaction rate in the dynamic biochemical systems. Clowes (1958) demonstrated that chlorinated phenols interfere with oxidative phosphorylation and the degree of interference is related to the stereochemical configuration of the phenol.

The question remains as to whether the influence of the phenols on the development enzymes is a reflection of alterations in oxidative processes. A more affininative answer to this problem would be obtained if a link were established between 
induced perturbations in the oxidative processes and the altered development times. Jdeally, to examine this possibility, one would choose a perturbation of a 'simple' nature, that is one created by non-chemical means and by the same token an alteration that the organism might conceivably encounter under the natural ontogenetic processes. As an example of such an interaction, oxygen availability to Drosophila pupae might be suddenly and drastically reduced by a temporary submergence under water. SzENT-GYORGYI (1957) points out that the withdrawal of oxygen from an organism will disorganize the oxidative system, and since oxygen is an electron acceptor such a withdrawal could conceivably have an effect similar to an antioxidant. Those isomers listed in Table 1 which induced the most pronounced increase in development time could also be acting in vivo as strong antioxidants. At least for one of these isomers this has been previously shown to be the case (EPSTEIN et al., 1965).

To examine this hypothesis concerning the influence of oxygen withdrawal, Drosophila pupae and pharate adults of known age (chronological age following formaticn of puparium at $23^{\circ} \mathrm{C}$ ) were submerged for brief intervals in water and their development time examined through nine inbred generations. The data listed in Table 2 demonstrate a significant increase in development time which is transferred to succeeding generations. Although the results of these experiments suggest

Table 2--Influence of Water submergence in the pupal and pharate adult stage on THE DEVElopment TIME of Drosophila

\begin{tabular}{ccc}
\hline Treatment & $\begin{array}{c}\text { Development time (days) } \\
\text { (mean of } 9 \text { inbred generations) }\end{array}$ & $\begin{array}{c}\text { Range } \\
\text { (days) }\end{array}$ \\
\hline $\begin{array}{l}\text { Control--no water treatment } \\
10 \text { min under } \mathrm{H}_{2} \mathrm{O}-\text { pupae } 88 \pm 8 \mathrm{hr}\end{array}$ & $13 \cdot 6$ & $11-16$ \\
$\quad$ (pharate adult ${ }^{*}$ ) & $17 \cdot 8$ & $15-20$ \\
10 min under $\mathrm{H}_{2} \mathrm{O}$ - pupae $56 \pm 10 \mathrm{hr}$ & $17 \cdot 3$ & $16-20$ \\
\hline
\end{tabular}

* See Hinton (1968).

that the inherited alteration in development time is brought about by reduced oxidation rates, at this point we can only infer that the phenols are also disrupting oxidative mechanisms. The in vivo sites of the perturbation or alterations in oxidative energy involved in the isomer studies may be quite different than those involved in the water submergence experiments. The data of Luning presented in Fig. 3 may also reflect drug-induced perturbations in the oxidative cycles. Here again, however, more studies will have to be made before these questions can be clearly resolved. These data certainly suggest, however, both in the isomer studies and in the submergence tests that altered rates of oxidation can influence development time and progeny in this organism.

\section{SUMMARY}

A consideration of the importance of the dihydroxybenzoic acid structure in the morphogenetic process of insect development led to an exploratory investigation 
of the influence of a series of these isomers on development patterns on $D$. melanogaster. Five dihydroxybenzoic acid isomers were added in a regular cornmeal molasses diet and the organisms were examined for a number of inbred generations. These isomers were shown to have a pronounced effect on the development time through the embryonic and postembryonic stages as well as on the yield of progeny. The degree of perturbation induced by a given isomer was shown to be related to its proton-donating power. The stereochemical configurations of the isomers indicated that they may be incorporated in the melanization process. The developmental alterations were suggestive of an enzyme inhibition mechanism and a plot of the data indicated an alteration in first-order reaction kinetics. The results from previously reported studies involving the influence of drugs on alterations in development time and progeny in Drosophila were also shown to agree with a proposed alteration in enzyme kinetics. A possible relationship between drug studies and the dihydroxy isomers is discussed.

From the fact that these isomers are known to act in vivo as antioxidants, the hypothesis was made that the isomers are inducing perturbations in the kinetics of oxidative processes. Additional evidence that the development time alterations are reflecting alterations in the oxidation processes was obtained by withdrawing oxygen from Drosophila pupae by means of brief submergence in water. This simple technique produced an increase in development timc which was shown to bc transferred to succeeding inbred generations. It is not known, however, whether the site of the perturbation is the same in the submergence tests as the centre of activity perturbed by the phenols.

\section{REFERENCES}

CheN Y. M. and Chavin W. (1966) Incorporation of carboxyl groups into melanin by skin tyrosinase. Nature, Lond. 210, 35-37.

Clowes G. H. A. (1958) The inhibition of cell division by substituted phenols with special reference to the metabolism of dividing cells. Ann. N.Y. acad. Sci. 51, 1409.

Epstein S. S., Saporoschetz I. B., Small M., Park W., and Mantel N. (1965) A simple bioassay for antioxidants based on protection of Tetrahymena pyriformis from the photodynamic toxicity of benzo(a)pyrene. Nature, Lond. 208, 655-658.

Gilmour D. (1961) The Biochemistry of Insects, p. 253. Academic Press, New York.

Hinton H. E. (1968) Spiracular gills. Adv. Insect Physiol. 5, 65-162.

Kyogoku Y., Lord R. C., and Rich A. (1968) Specific hydrogen bonding of barbiturates to adenine derivatives. Nature, I.ond. 218, 69-72.

LEVENGOOD W. C. (1965) Factors influencing biomagnetic environments during the solar cycle. Nature, Lond. 205, 465-470.

LevfrNgon W. C. (1966) Cytogenetic variations induced with a magnetic probe. Nature, Lond. 209, 1009-1013.

LeVENGOOD W. C. (1967) Morphogenesis as influenced by locally administered magnetic fields. Biophys. $\%$. 7, 297-307.

Levengood W. C. and Shinkle M. P. (1960) Environmental factors influencing progeny yields in Drosophila. Science, N.Y. 132,134-135.

LuNiNg K. G. (1966) Drosophila-test in pharmacology. Nature, Lond. 209, 84-86.

Richards A. G. (1953) Chemical and physical properties of cuticle. In Insect Physiology (Ed. by Roeder K. D.), pp. 32-34. John Wiley, New York. 
ShorTer J. and StubBs F. J. (1949) The additive effect of substituents on the strength of benzoic acid. F. chem. Soc. 1180-1183.

Szent-GyoRgyi A. (1957) Bioenergetics. Academic Press, New York. 